\title{
Erratum to: Along-slope oceanographic processes and sedimentary products around the Iberian margin
}

\author{
Francisco Javier Hernández-Molina • Nuno Serra • \\ Dorrik A. V. Stow • Estefanía Llave • Gemma Ercilla • \\ David Van Rooij
}

Published online: 10 November 2011

(C) Springer-Verlag 2011

Erratum to: Geo-Mar Lett

DOI 10.1007/s00367-011-0242-2

There is an error in the name of one co-author: Dorik A.V.

Stow should be Dorrik A.V. Stow (as shown above).

The online version of the original article can be found at http://dx.doi. org/10.1007/s00367-011-0242-2.

F. J. Hernández-Molina

Facultad de Ciencias del Mar, Universidad de Vigo,

36200 Vigo, Spain

N. Serra $(\bowtie)$

Institute of Oceanography, University of Hamburg,

Bundesstr. 53,

20146 Hamburg, Germany

e-mail: nuno.serra@zmaw.de

D. A. V. Stow

IPE-ECOSSE, Heriot-Watt University,

Edinburgh EH14 4AS, UK

E. Llave

Instituto Geológico y Minero de España,

Ríos Rosas 23,

28003 Madrid, Spain

G. Ercilla

Instituto de Ciencias del Mar, CMIMA-CSIC,

Paseo Marítimo de la Barcelonesa 37-49,

08003 Barcelona, Spain

D. Van Rooij

Renard Centre of Marine Geology (RCMG), Ghent University,

Krijgslaan $281 \mathrm{~S} 8$,

9000 Ghent, Belgium 